\title{
Harmonic Analysis of Power System Based on MATLAB
}

\author{
Hu Zhongyu ${ }^{1, a}$, Yue Qiang ${ }^{2, b}$,hesonglin ${ }^{1, c}$
}

${ }^{1}$ School of Auto-control and Mechanical Engineering, Kunming University, Kunming 650214,China

${ }^{2}$ School of Information and Technology, Kunming University, Kunming 650214,China

apoundblue@126.com, bwallay@126.com, ‘451061537@qq.com

Keywords: harmonic analysis, FFT window function, spectrum leakage

\begin{abstract}
This paper introduces the basic concepts of harmonic produced by MATLAB simulation, waveform of harmonic, harmonic of the fast Fourier transform to the spectral leakage phenomena are analyzed, introduced two kinds of solutions: increase the length of the window and select the appropriate window function. And the two kinds of solutions respectively. By simulation, several common window function in the inhibition spectrum of their respective characteristics.
\end{abstract}

\section{Introduction}

With all kinds of nonlinear loading, the wide application of power electronic equipment in power system especially, the harmonic pollution is becoming serious increasingly. Detection of harmonic components will be conducive to the power quality evaluation, and take the necessary measures to control. At present, the power harmonic analysis has become an important research direction in the field of power system. In recent thirty years, the rapid popularization of all kinds of electronic devices has harmonics pollution becoming more and more serious. Meanwhile the power quality declined. Therefore, real-time measurement of power and nonlinear harmonic components of electrical equipment and the actual situation in the palm grip system harmonic, which is necessary to prevent the harmonic harm and improve the quality of power supply [1-2].

\section{Basic Concepts of Harmonics}

In the power system, the normal voltage and current waveform should be sine wave with the power frequency, but the actual waveform is always distorted in different degrees. ${ }^{[3]}$ Especially in recent years, the converter in the distribution network, frequency control, electric arc furnace, electric railway, household electronic equipment and various power electronic devices is increasing, the load of the electrical characteristics (nonlinear, impact and asymmetry), make the power system voltage and current waveform distorting seriously. The distortion is periodic, which can be used in the Fourier series expansion is defined as follows: for periodic signal, when it satisfies the Dirichlet condition can be divided into the following series:

$$
\begin{aligned}
f(t) & =A_{0}+\sum_{n=1}^{\infty}\left[A_{n} \cos \left(n \omega_{1} t\right)+B_{n} \sin \left(n \omega_{1} t\right)\right] \\
& =A_{0}+\sum_{n=1}^{\infty} C_{n} \sin \left(n \omega_{1} t+\varphi_{n}\right)
\end{aligned}
$$

we define $A_{0}=\frac{1}{T} \int_{0}^{T} f(t) d t$, as direct-current component.

$A n=\frac{2}{T} \int_{0}^{T} f(t) \cos \left(n \omega_{1} t\right) d t, B n=\frac{2}{T} \int_{0}^{T} f(t) \sin \left(n \omega_{1} t\right) d t, \mathrm{Cn}=\sqrt{\mathrm{A}_{\mathrm{n}}^{2}+\mathrm{B}_{\mathrm{n}}^{2}}, \varphi_{n}=\arctan \frac{A_{n}}{B_{n}}$

$\varphi_{\mathrm{m}}$ is initial phase; $\omega 1=2 \pi / \mathrm{T} 1$, T1 is the fundamental component of signal fundamental period at $\mathrm{n}=1$, Sine wave components which's frequency is the fundamental integer multiples thereof of a series of are usually called harmonic, $\mathrm{n}$ is named the number of harmonic. According to the actual direct-current component equal to 0 and amplitude Is inversely proportional to frequency, when the harmonic frequency is too high for the entire periodic signal can be ignored, so the harmonic is generally expressed as shown in $\mathrm{Eq}(2)$. 


$$
x(t)=\sum_{n=1}^{N} C_{n} \sin \left(n \omega_{1} t+\varphi_{n}\right)
$$

The relationship between An- $\omega$ for amplitude spectrum, corresponding relationship with $\mathrm{n} \sim$ for the Omega phase spectrum.

\section{Harmonic Analysis In Time Domain Matlab}

MATLAB was introduced by Math Works, a technology application software. At present, MATLAB, because of its rich library, has become one of the software for computational science and engineering. The most popular international Matlab for harmonic analysis, the first problem of MATLAB is all of the variables are stored in the form of matrix, and the number of elements in the matrix and is inevitably limited, so that Matlab can only analyze the discrete signal. So the first step is the harmonic analysis Harmonic discretization, is sampled according to the Nyquist sampling theorem, the sampling frequency of FS is greater than 2 times the highest frequency signal, a continuous signal can be reduced without distortion. The harmonic signal after sampling is expressed as $\mathrm{Eq}(3)$ :

$$
x_{(m)}=\sum_{n=1}^{N} C n \sin \left(n \omega_{1} m+\varphi_{n}\right), \mathrm{m}=0, \frac{1}{f s}, \frac{2}{f s}, \ldots, \mathrm{M}
$$

The harmonic amplitude and initial phase angle is shown in table 1:

Table 1. parameters of harmonic

\begin{tabular}{|l|c|c|c|c|c|c|c|}
\hline $\begin{array}{r}\text { Harmonic } \\
\text { frequency }\end{array}$ & 1 & 2 & 3 & 4 & 5 & 6 & 7 \\
\hline $\begin{array}{l}\text { Angular } \\
\text { frequency (rad/s) }\end{array}$ & 50 & 100 & 150 & 200 & 250 & 300 & 350 \\
\hline Amplitude (V) & 5 & 1 & 3 & 1 & 2 & 1 & 1 \\
\hline $\begin{array}{l}\text { Initial phase angle } \\
\text { (DEG) }\end{array}$ & 10 & 15 & 30 & 35 & 50 & 10 & 20 \\
\hline
\end{tabular}

If $\mathrm{fs}=1250$, we can get the waveform of $\mathrm{X}(\mathrm{m})$ as shown in Fig.1.

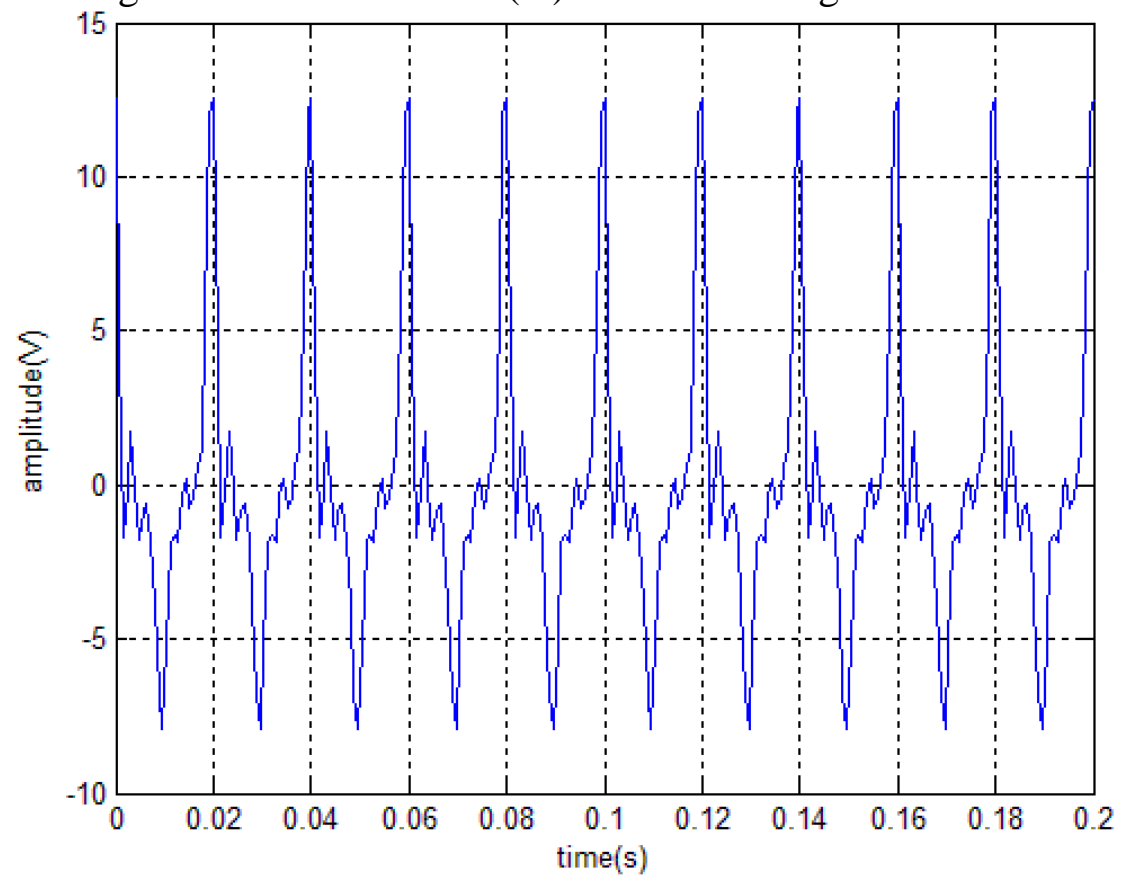

Fig. 1 time-domain waveform of X (m) 


\section{MATLAB Analysis of Harmonic}

At this time the value of $\mathrm{M}$ is limited brings two problems: first, whether the Fourier series or discrete $\mathrm{Fu}$ Liye transform, the time domain and frequency domain are infinite, so the discrete $\mathrm{Fu}$ Liye transform (DFT) is generally used to transform will be more accurate:

$$
X_{(k)}=\sum_{m=0}^{M-1} x_{(m)} e^{-j \frac{2 \pi}{M} m k} \mathrm{k}=0,1, \ldots, \mathrm{M}-1
$$

In fact, DFT speed is too slow, meanwhile we can only handle the sampling frequency of FS integer function, usually using fast Fourier transform (FFT). The results are the same, the calculation speed would be improved a lot[4-5].

The second is the leakage when harmonic signal is truncated. The spectrum will bring leakage on the main valve, We can reduced the leakage by increasing the number of $\mathrm{L}$ by, as shown in Figure 2, when $\mathrm{L}=0.1$, leakage of main lobe and side lobes are more serious, increasing the value of $\mathrm{L}$, when $\mathrm{L}=0.15$, the main valve leakage to improve the side lobe and almost no change, while the value of $\mathrm{L}$ is large enough, there is no leakage. But the real value of $\mathrm{L}$ in the bigger mean computation more computation time is longer, so the $\mathrm{L}$ can only be increased appropriately. The side lobe leakage need to select the window function better, window function start and at the end of the flat, the inhibitory effect of the side lobe will be better. The common window function as shown in Table 2

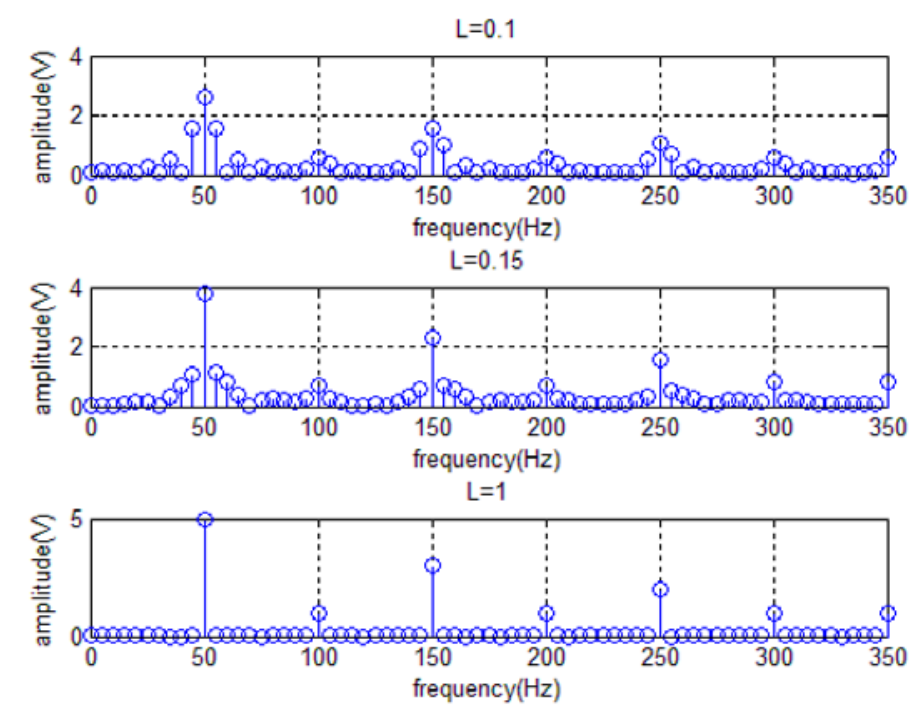

Fig. 2 Frequency-domain waveform of X (m)

Table 2 common window functions

\begin{tabular}{|c|c|c|}
\hline Name & Time- domain & Frequency-domain \\
\hline Rectangle & 1 & $\sin (\omega \mathrm{N} / 2) / \sin (\omega / 2) \cdot \mathrm{e}^{\mathrm{j} \omega(\mathrm{N}-1) / 2}$ \\
\hline hanning & $0.5-0.5 \cos \left(\frac{2 \pi \mathrm{n}}{\mathrm{N}}\right)$ & $\begin{array}{r}0.5 \mathrm{U}(\omega)+0.25\left[\mathrm{U}\left(\omega-\frac{2 \pi}{\mathrm{N}}\right)\right. \\
\left.+\mathrm{U}\left(\omega+\frac{2 \pi}{\mathrm{N}}\right)\right]\end{array}$ \\
\hline Hamming & $0.5-0.46 \cos \left(\frac{2 \pi \mathrm{n}}{\mathrm{N}}\right)$ & $\begin{array}{r}0.54 \mathrm{U}(\omega)+0.23\left[\mathrm{U}\left(\omega-\frac{2 \pi}{\mathrm{N}}\right)\right. \\
\left.+\mathrm{U}\left(\omega+\frac{2 \pi}{\mathrm{N}}\right)\right]\end{array}$ \\
\hline Blackman & $\begin{array}{l}0.42 \\
-0.5 \cos \left(\frac{2 \pi \mathrm{n}}{\mathrm{N}}\right) \\
+0.08 \cos \left(\frac{4 \pi \mathrm{n}}{\mathrm{N}}\right)\end{array}$ & $\begin{array}{r}0.42 \mathrm{U}(\omega)+0.25\left[\mathrm{U}\left(\omega-\frac{2 \pi}{\mathrm{N}}\right)\right. \\
\left.+\mathrm{U}\left(\omega+\frac{2 \pi}{\mathrm{N}}\right)\right] \\
+0.04\left[\mathrm{U}\left(\omega-\frac{4 \pi}{\mathrm{N}}\right)+\mathrm{U}\left(\omega+\frac{4 \pi}{\mathrm{N}}\right)\right]\end{array}$ \\
\hline
\end{tabular}


$\mathrm{L}=0.1$, for example, after multiplying harmonics with several common window functions, and then performing fast Fourier changes, as shown in Fig. 3.
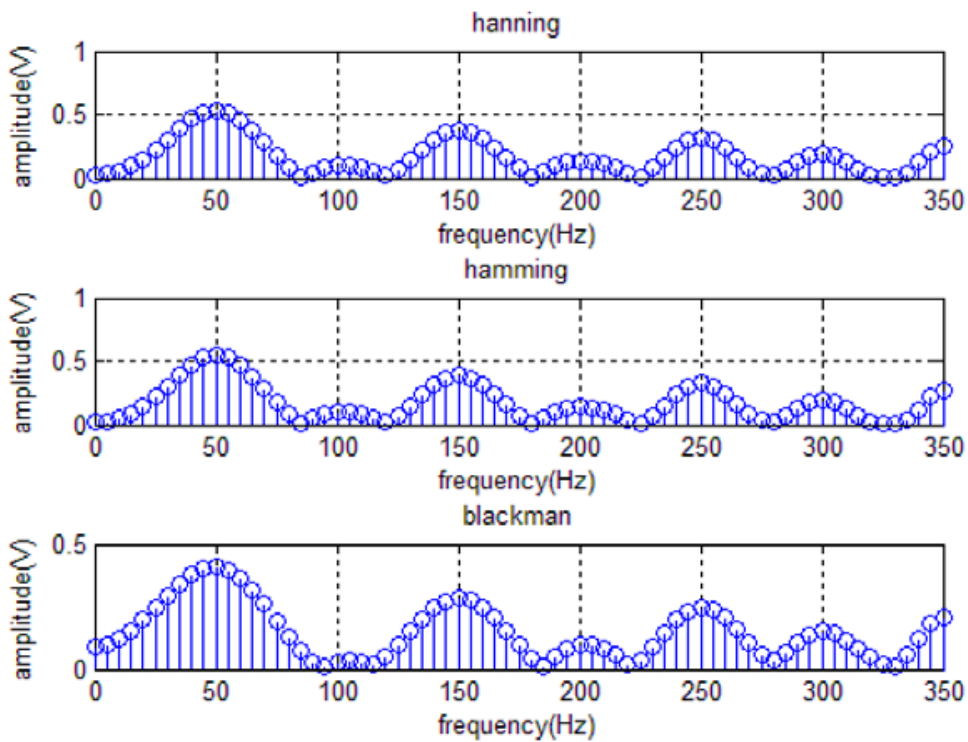

Fig. 3. suppression of side lobe leakage by three window functions

A rectangular window is shown in Fig 2, the harmonic side lobe window has almost suppressed to 0 , but at the same time the main lobe widening, lower frequency resolution, especially the main lobe Blackman window almost overlapped together, of course it's almost nothing. So the side lobe suppression and frequency resolution of vice flap improvement is a pair of irreconcilable contradictions, only according to the actual situation of judgment is important or the side lobe suppression resolution is important to select a suitable window function.

\section{Conclusion}

In this paper, the simulation and analysis by MATLAB of the harmonic signal of the time domain and frequency domain, to analyze the problems of spectrum leakage in frequency domain analysis, and explains the methods through simulation to solve spectrum leakage and several window functions for harmonic spectrum, a valuable role to play, open the door analysis of harmonic beginners into the field.

\section{References}

[1] Ignacio Santamaria-Caballero, Carlos J. Pantaleon-Prieto, JesusIbanez-Diaz, et al. Improved procedures for estimating amplitudes andphases of harmonics with application to vibration analysis [J]. IEEETransactions on Instrumentation and Measurement, 1998, 47(1): 209-214.

[2] Roberto Marcelo Hidalgo, Juana Graciela Fernandez, Raul RubenRivera, et al. A simple adjustable window algorithm to improve FFTmeasurements[J]. IEEE Transactions on Instrumentation and Measurement, 2002, 51(1): 31-36.

[3] Gregorio Andria, Mario Savino, Amerigo Trotta. Windows andinterpolation algorithms to improve electrical measurement accuracy[J]. IEEE Transactions on Instrumentation and Measurement, 1989,38(4):856-863.

[4] Pan Wen, Qian Yushou, Zhou E Power harmonics measurement based on windows and interpolated FFT (I) Study of windows Transactions of China Electrotechnical Society, 1994, 9(1):50-54.

[5] Pan Wen, Qian Yushou, Zhou E Power harmonics measurement based on windows and interpolated FFT (II) dual interpolated FFT algorithms Transactions of China Electrotechnical Society, 1994, 9(2):53-56. 